\title{
HACIA LA POSIBILIDAD DE UNA TEOLOGÍA POLÍTICA ECOFEMINISTA
}

\author{
Ely Orrego Torres
}

Northwestern University

Recibido: 09.07.2019 - Aceptado: 02.12.2019

\begin{abstract}
RESUMEN
En este artículo discuto una nueva aproximación al concepto de teología política. Mi propuesta es que al incluir la perspectiva ecofeminista se abren nuevas miradas para abordar la discusión de la teología política, que se ha caracterizado por una dominación geográfica, androcéntrica y antropocéntrica en el estado del arte sobre la teología política y su relación con la soberanía. El artículo se articula a partir de ideas planteadas por la teología ecofeminista de Ivone Gebara y Mary Judith Ress en el contexto latinoamericano. Para finalizar, planteo el caso del colectivo Con-spirando como una posibilidad política de acercarnos a nuevos modos de abordar el concepto de teología política.
\end{abstract}

Palabras clave: Teología Política; Ecofeminismo; Colectivo Con-spirando; Ivone Gebara; Mary Judith Ress.

\section{ABSTRACT}

In this article, I argue a new approach to the concept of political theology. I propose that a perspective that includes ecofeminist ideas can enrich and pluralize the state of the art in political theology. I suggest that a political theology inflected by ecofeminism can challenge geographic, androcentric, and anthropocentric biases that currently shape the debate on the relation between political theology and sovereignty. In what follows, I discuss ideas by ecofeminist theologians such as Ivone Gebara and Mary Judith Ress in the Latin American context. This article concludes with the case of the collective Conspirando as an exemplar of new possibilities of reshaping the concept of political theology.

Keywords: Political Theology; Ecofeminism; Collective Con-spirando; Ivone Gebara; Mary Judith Ress. 


\section{INTRODUCCIÓN}

La cuestión de la teología política es un tópico reciente en el estudio de la filosofía política, la teoría política y la ciencia política. Con el desarrollo de la filosofía continental y el pensamiento italiano contemporáneo, comenzó su auge intelectual a partir de los 90s, con las relecturas de Carl Schmitt y Walter Benjamin por parte de la academia internacional. Entre estas relecturas, un acontecimiento relevante que motiva su desarrollo es la publicación de la serie Homo sacer del italiano Giorgio Agamben1. Particularmente, la publicación de Homo sacer: Il potere sovrano e la nuda vita (1995) que planteaba una crítica a la idea de soberanía schmittiana. Por el otro lado, una reconocida revista internacional dedicada al tema como lo es Political Theology, comienza a ser editada a fines de los 90 y principios de los 2000.

En América Latina, la recepción de la teología política comenzó su crecimiento a fines de los 90' y se estableció de pleno en los 2000, con las lecturas de Jorge Dotti2 y Luis Oro3 sobre Schmitt; y la interpretación de Benjamin en conexión con la crítica de la cultura en el trabajo de filósofos chilenos como Nelly Richard4, Willy Thayer5, Pablo Oyarzún6 y Elizabeth Collinwood-Selby,7 quienes difundieron gran parte de sus ideas en la extinta Revista de Crítica Cultural.8 No obstante, será posterior a la llegada de las traducciones al español y difusión de la obra Homo sacer de Agamben,9 que la difusión de nuevos

1 Véase Agamben 1995, Agamben 1998, Agamben 2003, Agamben 2007, Agamben 2008 , Agamben 2011, Agamben 2012, Agamben 2014, Agamben 2015.

2 Véase Dotti 2000 y Dotti y Pinto 2002.

3 Véase Oro 2005 y Oro 2013.

4 Véase Richard 1994 y Richard 1999.

5 Véase Thayer 2007 y Thayer 2010.

6 Oyarzún ha sido uno de los traductores de Walter Benjamin al español, siendo su más famosa traducción La dialéctica en suspenso. Fragmentos sobre la historia publicada en 1999.

7 Véase Collingwood-Selby 1997 y Collingwood-Selby 2009

8 A partir de sus 18 años de existencia (1990-2008) y 36 números publicados, en palabras de Richard (2018), la revista se proponía "reunir aquellas escrituras críticas que se ubicaban en el filo de la academia haciéndolas transitar entre la universidad y sus afueras, con la motivación de estimular debates intelectuales sobre el rol de la cultura en el nuevo contexto de la reapertura democrática", ya que la crítica cultural designa "un dispositivo de análisis teórico, una operación de lectura y un registro de escritura que se ubican entre lo estético, lo político, lo ideológico y lo cultural".

9 En ese sentido, la labor de traducción y difusión de ideas de Edgardo Castro y Flavia Costa, ambos argentinos, ha sido relevante en el desarrollo de su pensamiento en la region. 
entendimientos de teología política cobrará más relevancia en la región.10 En el caso particular de Chile, los trabajos de Rodrigo Karmy11 y Miguel Vatter12 en relación con el concepto de biopolítica han aumentado el interés por las intersecciones entre ambas líneas de pensamiento. Otros esfuerzos intelectuales por visibilizar el debate crítico del concepto de teología política han sido expuestos en las revistas Deus Mortalis13 y Revista Pléyade14.

Como alguien que ha seguido de cerca el debate de la teología política, especialmente desde la llegada de los textos de Agamben, sugiero que el debate ha tendido a situarse en un eje de dominación geográfica y de género. Es decir, las corrientes principales son escritas y pensadas desde el hemisferio norte, o en función de autores pertenecientes a dicha región. Asimismo, los abordajes de la teología política tienden a ser androcéntricos y con escaso espacio para la interpretación desde una perspectiva de género. Asimismo, la deriva antropocéntrica de sus enunciados hace que surjan cuestionamientos sobre el lugar que la ecología u otros seres no humanos pueden ocupar en la cosmovisión de la teología política. Por tal motivo, una de las preguntas que surgen y motivan este artículo es sobre la posibilidad de plantear una teología política feminista, ecológica y desde el hemisferio sur. En ese sentido, ¿puede acaso la teología política expandir sus límites y abrirse a nuevos entendimientos de mundo y soberanía en un mundo post-secular?15 ¿De qué forma la perspectiva

10 Las traducciones al español han estado a cargo de Antonio Gimeno Cuspinera (en las ediciones españolas de Editorial Pre-textos); y de Flavia Costa, Edgardo Castro, Mercedes Ruvituso y Rodrigo Molina-Zavalía (en las ediciones argentinas de Editorial Adriana Hidalgo).

11 Véase Karmy 2011, Karmy 2014 y Karmy 2018.

12 Pese a que actualmente Miguel Vatter desarrolla su vida académica en Australia, durante su paso en Chile (2005-2011) impartió cursos en la Pontificia Universidad Católica de Chile y Universidad Diego Portales donde su foco de enseñanza e investigación fue la teología política y biopolítica. Hoy, se puede considerar uno de los principales exponentes en el mundo sobre la temática de teología política, especialmente, a partir de una lectura radical del concepto de soberanía. Para esas lecturas, véase por ejemplo Vatter 2004, Vatter 2011a, Vatter 2009, Vatter 2011 b, Vatter 2011c, Vatter 2012, Vatter 2013, Vatter 2016 y Vatter 2019a.

13 Jorge Dotti, quien era referente latinoamericano del pensamiento de Carl Schmitt, se desempeñaba como editor hasta su muerte en 2018.

14 Véase una de las ediciones pioneras sobre el tema en Chile y que fue publicada en 2011, mientras formaba parte del Centro de Análisis e Investigación Política (CAIP). En la introducción (Orrego 2011) y la entrevista a Vatter 2011c se desarrollan los argumentos que nos permiten entender cómo el concepto de teología política comienza a plantearse desde una perspectiva crítica a la soberanía y poder. La edición está disponible en: https://bit.ly/2XySldU En ese momento, la edición marcó un importante antecedente en el desarrollo de la temática como primera compilación en el país y región con voces reconocidas en el área y la academia. No obstante, hoy --casi diez años después--, tengo muchas autocríticas a esa primera edición. La ausencia de voces femeninas y de una mirada más enfocada en Latinoamérica son algunas de ellas. No obstante, en el corto camino intelectual que he recorrido, he podido reconocer y valorar otras voces que me han permitido un acercamiento distinto a las problemáticas planteadas por la teología política.

15 El término post-secular ha sido ampliamente debatido en la teoría política como una categoría que cuestiona la 'aparente' secularización en la época moderna. Algunos de sus expositores han sido Habermas 2008, Taylor 2007 y Casanova 1994. En líneas generales, se 
ecofeminista permitiría un abordaje diferente de lo que se ha entendido y planteado como teología política, particularmente, en lo que concierne a sus implicancias políticas?

Para desarrollar lo anterior, me enfocaré en el trabajo de mujeres teólogas ecofeministas cuyo trabajo se ha desarrollado en Latinoamérica, tales como Ivone Gebara y Mary Judith Ress, quienes han teorizado sobre nuevas posibilidades de vivenciar políticamente el cuerpo en comunidad con otras mujeres. Asimismo, se abordará el caso particular del grupo Con-spirando como una alternativa de resistencia y sororidad en un contexto de nuevas formas de espiritualidad y de hacer política.

\section{EL CUERPO DE LAS MUJERES Y EL MAL RADICAL}

Cuando en 1994 la teóloga brasileña y ecofeminista Ivone Gebara hace unas declaraciones sobre el aborto, jamás pensó que su "castigo" sería volver a estudiar teología y guardar silencio por un tiempo. Gebara -quien había sido influenciada por la teología de la liberación y denunciaba injusticias de género desde la teología feminista-, no solo fue censurada por el Vaticano, sino que tuvo que repetir sus estudios de doctorado en la Universidad Católica de Lovaina y asumir el silencio obsequioso con el cual fue castigada.

Su respuesta a dicho período de censura y silencio se materializó en su tesis de doctorado titulada: "Rompiendo el silencio: una fenomenología feminista

trataría de un concepto que alude a repensar las categorías de religión y política, considerando que la religión no ha sido abolida de la esfera pública, como el liberalismo habría planteado. Sobre un posicionamiento crítico desde las nociones de biopolítica y pensamiento politico italiano, véase el reciente artículo de Vatter 2019b.

Pese a estas consideraciones iniciales, mi posicionamiento está alineado con lo propuesto por Saba Mahmood 2005 y Joan Wallach Scott 2018. En el caso de Mahmood, a partir de su análisis del movimiento pietista en Egipto, desarrolla un análisis del feminismo en el contexto del Islam. A partir de ello, plantea un argumento polémico sobre qué significaría la libertad femenina en un contexto no-Occidental y asimilado principalmente como "patriarchal" y carente de derechos hacia las mujeres. En el caso de Scott, hace una crítica al concepto de 'secularización'. En sus palabras, el término 'secularización' nunca consideró la equidad de género ni derechos de las mujeres, sino que ha perpetuado el poder de Occidente y la dominancia del hombre blanco Cristiano. Aunque ambas no utilizan el término post-secular, me parece interesante el abordaje de la problemática del liberalismo como una falsa utopía de igualdad en derechos, especialmente cuando se trata de la supremacía de una religión (cristianismo) con una vision particular (occidental, androcéntrica y antropocéntrica) en contraste con otras religiones, por ejemplo, en el caso del Islam que ambas autoras plantean. De este modo, mi abordaje a la problemática no tiene que ver solo con la pregunta de incluir o no lo religioso en la esfera pública, sino que a qué tipo de abordaje nos referimos con ello. En este artículo planteo que una mirada política tendría que incluir cuestionamientos a determinada idea de cristianismo, así como una alternativa argumentativa que debata un posicionamiento desde otros lugares geográficos y de género. Es decir, nuevos modos de acercarse a la crítica de lo que se ha planteado hasta ahora como teología política. 
del mal"16, siendo considerada una de sus mayores obras. En dicho texto, ofrece una perspectiva innovadora sobre el problema de Dios y el problema del mal visto desde las mujeres. En ese sentido, "no solo el mal que cada uno comete personalmente, sino también el mal padecido, el mal soportado y no elegido, el mal presente en determinadas instituciones y estructuras culturales y sociales que lo favorecen" (Gebara 2002, 17). En otras palabras, Gebara respondía a lo que significó su "castigo" desde el Vaticano, pero además, era una posibilidad de hablar desde la experiencia de las mujeres y la opresión de sus cuerpos.

En la teoría feminista, el rol que se le atribuye al cuerpo es clave para entender el significado de la dominación patriarcal en la sociedad. En este sentido, la teología política también se ha enfocado en un análisis de la función de los cuerpos y su vinculación con la soberanía. En 1995, Giorgio Agamben planteaba la noción de homo sacer a partir del poder ejercido sobre el cuerpo: "El cuerpo mismo del homo sacer, en su condición de insacrificable al que, sin embargo, se puede matar, es la prenda viviente de su sujeción a un poder mortal, que no consiste, sin embargo, en el cumplimiento de un voto, sino que es absoluta e incondicionada. La vida sagrada es vida consagrada sin que sea posible ningún sacrificio y más allá de cualquier cumplimiento" (Agamben 1998, 129). A partir de lo anterior, la condición de homo sacer está condicionada por esta ambivalencia entre lo sacrificable y lo no sacrificable, 17 así como por su consagración a los dioses y el poder mortal sobre su cuerpo. Es por ello que la sacralidad de la vida y la relación con la teoría de la soberanía, basándose en la interpretación de los dos cuerpos del rey de Ernst Kantorowitz,18 condicionará la perspectiva teológico política de Agamben y su obra.

Sin embargo, ¿cuál es el rol del cuerpo en el caso de la teología feminista? ¿de qué forma se busca reinterpretar el cuerpo de las mujeres desde la espiritualidad e instituciones religiosas? En primer lugar, la teología feminista ha encontrado su lugar en las imágenes y la resignificación de lo divino. La pregunta sobre Dios padre y la deconstrucción del mismo, así como de la tradición judeocristiana en su conjunto, han sido temas constantes en su discusión.19

Desde antaño, la mujer ha cargado con el mito de Eva y su culpabilidad, además de la invisibilización de otras mujeres en la historia bíblica, a excepción de la figura de la Virgen María. La mujer ha sido catalogada como fuente de lujuria, tentación y pecaminosidad para los hombres, conllevando una distinción

16 En español ha sido publicada bajo el nombre de El rostro oculto del mal: una teología desde la experiencia de las mujeres (2002).

17 Sobre la relación entre lo insacrificable y la violencia divina, véase Orrego 2008.

18 Sobre ello, véase el capítulo 5 de la segunda parte de Homo sacer. Il potere sovrano e la nuda vita (1995).

19 En particular, recomiendo la excelente antología editada por Mary Judith Ress, Ute Seibert y Lene Sjørup titulada Del cielo a la tierra (1994). En particular, véanse los artículos de las partes 2 (La crítica clásica), 3 (La diosa) y 4 (¿Qué hacemos con la tradición judeo-cristiana?). 
primordial en cómo se ha tratado el tema del mal en la teología y el sacrificio realizado por ella. Como menciona Gebara, este vivir en base al sacrificio de obedecer al Dios padre, se ha traducido en la obediencia a los hombres que ostentan un cierto poder social y religioso, tales como el marido, los hermanos, los sacerdotes (Gebara 2002, 120). El problema no sería solo esta obediencia absoluta a la figura masculina desde nuestra infancia, sino el cómo la vida de las mujeres ha significado una renuncia al placer, propios pensamientos, sueños, propia voluntad, es decir, una renuncia a su cuerpo para ponerse al servicio de los demás o vivir según lo que dicen otros sobre ella (ibid.).

A partir de este sacrificio y potencial culpabilidad de las mujeres por no cumplir los cánones esperados, las mujeres asumirían un comportamiento de soportar y aceptar el sufrimiento como parte del miedo que se reproduce desde nuestra infancia. La misma experiencia de Ivone Gebara con los testimonios de mujeres manifiesta una expresión del mal femenino en el 'no tener', 'no saber', 'no poder', 'no valer', o en el 'mal del color de la piel'20 como una "interiorización del mal que me afecta como dependiente, hasta cierto punto, de mi responsabilidad" (Gebara 2002, 124). De este modo, podemos afirmar que el cuerpo mismo de las mujeres se ve sometido a violencias culturales y físicas por su condición de ser mujer. Y con el tiempo, las mujeres asumen esa posición y reproducen ese mal, especialmente, en el espacio doméstico. Como afirma Gebara, son ellas las que reproducirán el modelo patriarcal en las estructuras sociales más fundamentales como es el hogar, la escuela y la iglesia (ibid., 133). Igualmente, sucede una lógica similar en el ámbito público con la entrada de mujeres a un espacio principalmente masculino y donde actuarían con opresión hacia otras, por medio de una reproducción del patriarcado.

Aunque el llamado 'mal de las mujeres' denunciado por Gebara es una realidad que viven las mujeres, sean cristianas o no, la deconstrucción de la imagen de lo divino y de los propios cuerpos otorgarían una respuesta a cómo vivir de otro modo posible. Según la teología feminista, uno de los grandes descubrimientos de las mujeres que experimentan su espiritualidad desde el

20 A partir de una serie de testimonios, Gebara desarrolla su entendimiento del mal femenino como a) no tener, basado en la responsabilidad de la mujer de alimentar a la familia y de la responsabilidad de cuidado y educación. Por ejemplo, la prostitución como una profesión para vivir y hacer vivir; b) no poder, basado en la experiencia de la pobreza que trasciende a mujeres de todo el mundo y en cómo se reprimen las necesidades vitales del cuerpo: cuerpo condenado por el hambre y sed, cuerpo condenado por la falta de vivienda, cuerpo condenado por la enfermedad, cuerpo golpeado, cuerpo expuesto a la violencia; c) no saber, basado en la educación negada a las mujeres, un saber prohibido en su tiempo y condición, donde la educación y sabiduría era un espacio eminentemente masculino; d) no valer, llamado el lugar de crucifixión de las mujeres, ya que no solo refiere al valor que se le atribuye a las mujeres en relación con los hombres, sino que con otras mujeres, entendidas como 'objetos' de placer, odio, venganza; e) el color de la piel, siendo una de las fuentes de dolor para las mujeres en América Latina, de ascendencia africana, ya que es un dolor impuesto por una cultura que establece una jerarquía de las personas a partir del color de la piel (Gebara 2002, 39-65). 
feminismo es superar la figura del Dios masculino judeo-cristiano y abrir paso a un amor hacia lo divino dentro de ellas mismas. En ese sentido, significa abrirse al simbolismo de la Diosa21 como afirmación del poder de la mujer, del cuerpo de la mujer, de la voluntad de la mujer y de los vínculos y herencia de las mujeres. O como se pregunta Carol P. Christ: "la dimensión espiritual del feminismo ¿es una diversión pasajera, un escape del difícil pero necesario trabajo político? ¿o este surgimiento del símbolo de la Diosa entre las mujeres tendrá implicancias políticas y sicológicas significativas para el movimiento feminista?" (1994, 159). En otras palabras, lo que plantearon las teólogas feministas en ese entonces, no solo remite a una reinterpretación de las lecturas bíblicas, sino que también a nuevos modos de entendimiento de lo divino que tendrían consecuencias en su accionar y comprensión de ser mujer en el contexto de la Iglesia cristiana.

Con la divulgación del cristianismo, las figuras femeninas han tomado un rol secundario. Un caso excepcional ha sido María, en tanto madre del Mesías y virgen, pero que adquiere su importancia por su rol de madre y el extraordinario don de concebir sin pecado. No obstante, de otras mujeres discípulas como María Magdalena o Marta conocemos lo que la tradición se ha encomendado difundir: María Magdalena como una prostituta y pecadora, pero que halla la salvación; Marta como la anfitriona de la casa y quien se muestra afanada por las labores domésticas en presencia de Jesús (Lc. 10.38-41). Esa invisibilización les habría costado a las mujeres el sometimiento de sus cuerpos y una "disposición de ánimo de confianza en el poder salvífico masculino y de desconfianza en el poder femenino en ella misma y las demás mujeres, considerándolo inferior y peligroso" (Christ 1994, 161). En ese sentido, es una disposición que se transformaría en una motivación que se convierte en una realidad social y política, especialmente en las instituciones y esfera pública.22 Por ello, la significación de la Diosa en las mujeres contempla una afirmación de sus cuerpos ya que es de donde emana el ciclo de la vida, a diferencia de los tabúes con los que las mujeres hemos cargado en vida como la menstruación, alumbramiento y menopausia (ibid., 165). Lo interesante de esta imagen de la Diosa es que no necesita de una imagen exterior, ni de un templo ni de dogmas bajo los cuales se ha construido mucho de la tradición cristiana, sino que la Diosa está en cada mujer, la vive y concibe como tal en tanto experimenta una conexión con su cuerpo y con otras en comunidad.

21 Existen relatos de la creación del mundo que aluden a deidades femeninas en el Cercano y Medio Oriente. Así como en las civilizaciones antiguas que hasta el día de hoy guardan vestigios de templos dedicados a las diosas de la tierra, fertilidad, entre otras. Una de las más emblemáticas es Ashtoreth, considerada una diosa "pagana" en el Antiguo Testamento, pero que se le conocía en el Canaán como la Reina del Cielo. Véase Stone 1994.

${ }_{22}$ Como una respuesta a esta "deuda histórica" con las figuras femeninas en el temprano cristianismo, véase el reciente libro de Kateusz (2019), enfocado en una nueva interpretación histórica de María y otras mujeres. 
De una u otra forma, esta visión de teología feminista quiebra con los fundamentos de una teología política tradicional planteada desde la noción del soberano, legitimando el concepto de autoridad y jerarquía característico de las religiones monoteístas. Aunque este es un primer acercamiento a lo que podría entenderse como una nueva forma de teología política desde el feminismo, es necesario introducir el concepto de ecofeminismo y su deriva desde la resistencia para entender su potencialidad en una comprensión post-secular.

\section{RESPUESTA ECOFEMINISTA EN UN MUNDO POST-SECULAR}

Las teologías críticas de América Latina que se comenzaron a desarrollar a partir de los 60' conllevaron una evolución desde ese entonces hasta la actualidad. Como fruto de una nueva forma de pensar una teología en diálogo con la realidad latinoamericana y la opción por los pobres, surge la teología de la liberación de la mano de teólogos como Gustavo Gutiérrez, Leonardo Boff y Rubem Alves. En ese contexto de crítica a la autoridad eclesial y basándose en un cristianismo de bases, nace la teología feminista en Latinoamérica con representantes como Elsa Tamez, Ivone Gebara y Mary Judith Ress. Su desarrollo puede catalogarse en tres etapas: una primera etapa (años 70') con la identificación de mujeres biblistas y teólogas con los métodos y prácticas de la teología de la liberación donde se veían a sí mismas y a otras mujeres como sujetos de la historia y protagonistas de la liberación. Una segunda etapa (años 80') con una consciencia creciente en que la teología de la liberación contenía una mentalidad patriarcal que las incomodaba. Por lo que comenzaron a producir recursos teológicos de forma creativa expresados en liturgia, arte y poesía, además de comenzar a relacionarse con otros movimientos de mujeres de la región y con teólogas feministas del Primer Mundo. Y finalmente, una tercera etapa (años 90' al presente) que desafía el modelo de antropología patriarcal y la cosmología de la teología de la liberación pidiendo una reconstrucción de la teología desde la perspectiva feminista. En otras palabras, lo que Ivone Gebara ha llamado un "ecofeminismo holístico" (Ress 2012, 15-20).

A partir de esta noción de ecofeminismo holístico es que trazaremos alternativas para comprender lo que se entendería como un nuevo entendimiento de teología política en un mundo post-secular. Este concepto de ecofeminismo holístico ha significado una reestructuración muy radical -e incluso postcristiana-, no obstante, con mucho significado para las mujeres pobres latinoamericanas con sentido de pasión y alegría (ibid., 120). No obstante, ¿cómo surge esta deriva crítica de una interpretación crítica que en sí misma constituía la teología de la liberación? Según Gebara, pese a que la teología de la liberación cuestionaba el hambre, injusticia, dictaduras y destrucción de pueblos enteros en la América Latina de los años 60' y 70', ésta no había desafiado la antropología y 
cosmología patriarcal del cristianismo (ibid., 121). Sumado a la situación de las dictaduras en la región, el ecofeminismo surge como una respuesta a movimientos feministas y ecológicos que comienzan a desarrollarse en el mundo.23 Tales movimientos comenzarían a desafiar la llamada "hegemonía del imperio patriarcal" (Gebara 2000,17), aunque existen intelectuales y mujeres que se oponen a relacionar feminismo con ecología como luchas comunes.

Pero, ¿qué es el ecofeminismo? En palabras de Gebara:

"El ecofeminismo como pensamiento y movimiento social refiere básicamente a la conexión ideológica entre la explotación de la naturaleza y la explotación de las mujeres dentro del sistema jerárquico-patriarcal. Desde el punto de vista filosófico y teológico, el ecofeminismo puede ser considerado como una sabiduría relegadas por el sistema patriarcal, y particularmente por la modernidad, a ser fuerza de reproducción de mano de obra -"vientres benditos"- en tanto la naturaleza se tornó objeto de dominación para el crecimiento del capital. Como recuerda bien Carolyn Merchant, la modernidad -aunque los historiadores no hablen de esocomienza con la tortura de las brujas y el establecimiento de un nuevo método científico. Las brujas fueron consideradas no sólo símbolos del mal, sino también de la violencia de la naturaleza, capaz de provocar tempestades y enfermedades, de matar niños. La asociación entre las mujeres y la naturaleza era clara. Por eso las mujeres revoltosas y la naturaleza en desorden precisaban ser controladas" (ibid., 18).

De la cita anterior, podemos rescatar diversos elementos que nos permitirán esbozar los lineamientos del ecofeminismo holístico. En primer lugar, la base del pensamiento ecofeminista que está en relación con una cosmología ligada a la naturaleza. No es casual que en el ecofeminismo se aluda a la Madre Tierra como aquella madre -e incluso diosa- cuya conexión trasciende lo terrenal, alcanzando una nueva espiritualidad. En esa línea, el ecofeminismo cuestiona también la racionalidad moderna, cuya expresión ha sido el capitalismo que oprime no solo a la humanidad, sino que también a la misma naturaleza, es decir, a la madre. Por ello es que la misma crítica que realiza Gebara a la teología de la liberación alude a la reconsideración de una cosmología holística. Es decir, no basta con una crítica al capitalismo y una opción por los pobres si no existe una crítica misma al androcentrismo y antropocentrismo inherentes al sistema en cuestión. Y por último, nos remite a lo discutido en la sección anterior sobre el "mal de las mujeres" y su expresión en las brujas, donde éstas eran responsables de los daños provocados por la naturaleza, debido a su íntima conexión. Dicho esto, una vez

23 Según Mies y Shiva, el concepto ecofeminismo remite a "un nuevo término para una sabiduría antigua" que se introdujo a fines de los años 70' y principios de los 80 '. Francoise D'Eaubonne lo utiliza por primera vez en el contexto de los movimientos sociales y las protestas en contra de los desastres medioambientales de la época $(2014,13)$. 
más se confirma lo que Gebara planteaba sobre la culpabilidad de las mujeres como responsables del mal en el mundo.

En este punto es relevante destacar la especificidad del pensamiento ecofeminista. A diferencia de la teología de la liberación que tenía como objeto la preferencia por los pobres como una condición socioeconómica, el ecofeminismo alude a la triple discriminación que significa ser mujer en un contexto latinoamericano: discriminación por género, clase y raza24. Y aunque su énfasis es la condición de ser mujer, se insiste en que la liberación y nueva comprensión tiene que darse equitativamente en hombres y mujeres dentro del cosmos para reconstruir las nociones sobre divinidad. En ese sentido, re-situar a los individuos dentro -no por encima- del universo y clamar por una nueva relacionalidad que, en palabras de Gebara, "es la realidad básica de todo lo que es o puede existir. Es el tejido subyacente que está en continuo movimiento dentro del proceso vital en el cual estamos inmersas" (Gebara en Ress 2012, 123). En otras palabras, se abre una posibilidad ética de abrir espacios de relación en la humanidad y con la naturaleza.

Esta respuesta ética se opondría a la noción de jerarquía de poder que nos ha enseñado el cristianismo sobre la relación individual con lo divino. Es por eso que la mujer y la naturaleza siempre se han planteado desde una visión de sometimiento y dominación que comienza en el Génesis, donde el hombre racional está por sobre el mundo. En otras palabras, se plantea el hombre como un dios. Llama la atención que esta noción de poder del hombre se visibiliza como un poder de sometimiento a otro, en tanto es un poder exterior y que necesita ejemplificarse con las jerarquías y autoridad, es decir, con la necesidad de manifestar esa soberanía. No obstante, cuando nos referimos a la relación de poder en el ecofeminismo, encontramos una noción que no requiere de tal manifestación. Según Primavesi, al hablar de ecofeminismo y ecología, encontramos otras formas de poder: poder-del-interior y poder-con, reuniendo espíritu y cuerpo, humanidad y naturaleza, Dios y mundo $(1994,478)$. De este modo, no se necesitaría de un soberano o soberana para su posibilidad, sino que el relacionamiento consigo mismo, con otros y con la naturaleza. De este modo, retomamos la importancia que adquiere el cuerpo personal y su conexión con la tierra. Al decir que existe un poder-del-interior, éste en muchas oportunidades se desconoce o se reprime por las mismas mujeres. Forma parte de una espiritualidad que necesita de la conexión con la tierra y con el significado del cuerpo para las mujeres que está dado por el ciclo natural de la vida. Una de sus expresiones es por medio de las danzas y ritos que permiten a las mujeres conectarse con ese aspecto poco explorado. Hablar desde esta espiritualidad significa aflojar la Diosa que está dentro de cada una por medio de la creación, símbolos y rituales. Sin embargo, es un camino que se hace en comunidad con

24 Sobre los aportes de Ivone Gebara a la teología de la liberación, véase van Andel 2014. 
otras, donde mujeres pueden relacionarse y compartir experiencias, así como nuevas espiritualidades. Un buen ejemplo de ello y de cómo ha influido el ecofeminismo en las mujeres latinoamericanas es el Colectivo Con-spirando que surge como una alternativa y posibilidad de resistencia a las teologías políticas tradicionales.

\section{COLECTIVO CON-SPIRANDO: ALTERNATIVA ECOFEMINISTA EN UN MUNDO POST-SECULAR}

El colectivo Con-spirando nace a principios de los 90' en Chile como un espacio alternativo de mujeres feministas en busca de nuevas visiones en los ámbitos de espiritualidad, teología feminista y ecofeminismo.25 Desde sus inicios las ha convocado la política, el universo, el cuerpo, la cultura y la vida cotidiana. Se consideran parte del movimiento feminista y otros movimientos sociales.26

En palabras de Gebara, hay cinco contribuciones del colectivo al desarrollo de las mujeres y una nueva espiritualidad: "1. La fe está en la sabiduría de nuestros cuerpos y la prioridad de conocer a través de nuestra corporalidad en relación (...); 2. Los esfuerzos para buscar modos no-jerárquicos de ser que modelen el 'poder con' en lugar del 'poder sobre'; 3. El compartir nuevas formas de celebrar, nuevos rituales que nutren nuestras espiritualidades emergentes y nuestros compromisos; 4. El reexaminar aquellos mitos fundacionales en los cuales la cultura cristiana occidental está basada, para poder revitalizarlos y buscar nuevos mitos que puedan alimentar nuestras espiritualidades, teologías y éticas emergentes; 5. Todas nosotras somos una. Todos son mis familiares, desde las personas del barrio a los animales, a las montañas, a los ríos" (Ress 2012, 131132).

En entrevista con Mary Judith Ress, una de sus fundadoras, me comentaba que entre sus motivaciones estaban el conocerse desde el cuerpo, cambiar la epistemología imperante en la tradición jerárquica y patriarcal cristiana, así como empoderarse como mujeres por medio de la celebración. Este último aspecto es relevante, porque la causa de reencontrarse como mujeres era cambiar el concepto de mal que se les había atribuido desde antaño y en vez de responder desde la rabia, debía hacerse por medio de la celebración. Esta celebración no implicaba cambiar de fe ni de iglesias, a las que la mayoría de las mujeres participantes iban, sino que otorgar resignificaciones por medio de ritos referidos a los ciclos de la naturaleza, las estaciones del año y reencuentros con las

25 De acuerdo a Mary Judith Ress, las mujeres que participaron en esos años del colectivo estaban fuertemente influenciadas por la teología de la liberación. Posteriormente, comenzarían a identificarse como "ecofeministas" (Ress 2006, xi).

26 Para más información sobre el colectivo, puede visitarse su página web: www.conspirando.cl 
hermanas. En ese sentido, Con-spirando comienza a constituirse como un espacio de resistencia y alternativa a los ritos que se les ofrecían en sus espacios eclesiales. Si en el templo se les prohibía danzar, en los ritos danzaban; si en las liturgias existían estructuras jerárquicas y establecidas, en los ritos no había estructuras y se danzaba en círculos; si en la iglesia no se hablaba de la Diosa, en los ritos se comienza a celebrarla. Es decir, estos espacios significaron lo que las mujeres anhelaban y soñaban de un lugar de espiritualidad y dándole voz y sentido a las mujeres. Sin saberlo, estaban rompiendo con el sistema sin saber que lo hacían.

$\mathrm{Y}$ ese romper con el sistema implica un cambio en la cultura y relación con el cuerpo, como se planteó en la segunda parte de este artículo. Así como no hay liberación sin un cambio de paradigma. Por ello el trabajo de Con-spirando ha significado denunciar la violencia teológica hacia las mujeres, renombrando y resignificando lo sagrado, desarrollando nuevas metodologías y trabajo con el cuerpo, así como la contribución teológica ecofeminista mediante la revista Conspirando que llegó a publicar sesenta ediciones.

En ese sentido, me parece pertinente aludir a la propuesta pedagógica que un colectivo como Con-spirando aporta a nuevos entendimientos de una teología política crítica hoy. Autoras feministas como Saba Mahmood (2005) y Chandra Talpade Mohanty (2003) han cuestionado la categoría de "mujer tercermundista", principalmente por la idea de que estas serían ignorantes, pobres, religiosas y orientadas a la familia (Mohanty 2003, 40). No obstante, el cómo se han logrado constituir y formar comunidades basadas en solidaridad y prácticas religiosas son aspectos que tanto Mohanty como Mahmood han destacado de sus investigaciones con mujeres pertenecientes al llamado "Sur global" o "Dos-Tercios" del mundo.27 En ese sentido, ambas destacan el rol de una pedagogía crítica, basada en el repensar la posición de ser mujer en el mundo y en el contexto particular. En el caso de Mahmood, ella destaca la importancia de los rituales en el Islam y las posturas del cuerpo, siendo un espacio de aprendizaje con otras mujeres (2005, cap. 3 y 4). Este aprendizaje permitiría abrir espacios para enlazar conocimiento, responsabilidad social y lucha colectiva, con el fin de desafiar los espacios de dominación y crear espacios públicos más equitativos (Mohanty 2005, 201). Pese a que Mohanty realiza su propuesta en el contexto de las universidades y la academia norteamericana, su análisis es

27 Utilizo el concepto "Sur global” como una expresión geográfica, pero también histórica y con implicancias comunes, por ejemplo, la colonización e imposición del cristianismo. No obstante, es un concepto complejo, ya que la misma Mohanty ha concebido que en vez de hablar de Primer Mundo y Tercer Mundo o Norte y Sur, se proponga la nomenclatura Un-Tercio y DosTercios (One-Third/Two-Thirds), ya que no se plantea como conformada por conceptos opuestos, sino que como puntos de conexión entre las comunidades de mujeres marginadas y privilegiadas a nivel local y global (Mohanty 2005, 243). 
pertinente para el caso de una pedagogía crítica, horizontal y que cuestiona las nociones de autoridad y lo divino, como ha planteado Con-spirando.

En ese sentido, históricamente, la mujer latinoamericana ha lidiado con las nociones peyorativas de ser mujer, especialmente, por una "aparente" religiosidad y ausencia de cuestionamiento a las autoridades eclesiásticas. Sin embargo, la propuesta de Con-spirando desafía estas nociones. En primer lugar, cuestiona la teología de la liberación como androcéntrica y antropocéntrica. En segundo lugar, replantea una cosmovisión de lo divino en tanto excluye a las mujeres y el cosmos de la plena participación del cuerpo soberano. En otras palabras, la deconstrucción de lo divino propuesto por Con-spirando y las teologías ecofeministas refieren a reinterpretar el cuerpo de las mujeres. Y junto con esa reinterpretación del cuerpo, también aluden a un cuestionamiento del concepto de poder. Como ha planteado la noción de soberanía teológico-política, el 'poder sobre' ha determinado la forma de hacer política. No obstante, el 'poder dentro' desafía esa noción de soberanía situándose en el cuerpo de cada individuo y resignificándolo en cómo se construyen caminos comunes y colectividades basadas en solidaridades y sororidades en el espacio religioso.

A pesar de esas aportaciones, no hay dudas de que el mayor impacto se ha expresado en los testimonios de las muchas mujeres en Latinoamérica que han sido tocadas, animadas y empoderadas por el colectivo Con-spirando28. No solo hallando un espacio de acogida y alegría, sino que también de lamento y compartir tristezas. Incluso, soñando nuevos mundos posibles y articulándose políticamente en los nuevos movimientos feministas que se están configurando hoy en Chile. Y a pesar de que sus fundadoras temen acerca de la renovación del colectivo en el futuro, escriben con esperanza: "En lugar del predominante 'poder sobre', la semilla sugiere 'el poder dentro'. La semilla permanece dormida, se rompe, brota, florece, da fruto, madura, se seca y cae a la tierra de nuevo. Será lo que deba ser. Nosotras también somos semillas, llamadas a ser lo que debemos ser. Y lo mismo es Con-spirando. Que sea lo que tenga que ser -nada más ni nada menos" (Ress 2012, 144).

\section{REFLEXIONES FINALES}

Cuando se me planteó la posibilidad de escribir sobre teología política, inmediatamente surgieron en mi cabeza los conceptos de soberanía, autoridad y jerarquía. Y es que Schmitt no estaba tan errado cuando establece que "todos los conceptos centrales de la moderna teoría del Estado son conceptos teológicos secularizados" (2009, 37). Sin embargo, me inquietaba la idea de presentar la soberanía o 'poder sobre' como una categoría incuestionable. Así como en la

28 En el capítulo 4 del libro de Mary Judith Ress (2012) se plantea una serie de reflexiones a partir de las experiencias de mujeres latinoamericanas en relación con el ecofeminismo. 
teología encontramos fundamentos para la dominación y violencia, también hallamos su posibilidad de redención y liberación de los oprimidos. Y al hablar de oprimidos, a diferencia de la teología de la liberación, que lo hace exclusivamente desde la condición socioeconómica, me sitúo en aquellos y aquellas que sufren exclusión y opresión por su género, raza, casta y otras formas de discriminación en la actualidad. Incluso, podríamos hablar de dominación sobre la especie y la naturaleza, como lo plantea la teología ecofeminista.

La configuración de nuevos entendimientos de relacionamiento entre seres humanos y hacia la naturaleza son claves para dar significado a la comprensión de teología política en la actualidad. Los desafíos refieren a vivir el ecofeminismo no exclusivamente en la esfera privada de la espiritualidad, sino como un modo de vivir políticamente. El ecofeminismo nos invita a cambiar el paradigma de cómo estamos relacionándonos no solo con el otro ser humano, sino que con la naturaleza. Y hoy, en que las noticias nos alertan sobre el cambio climático y las consecuencias de la era del antropoceno -que refleja el impacto del humano en la tierra- y la era tecnozoica -enfocada en la explotación de los recursos y del planeta por medio de la tecnociencia-, cada vez se hace más difícil avanzar hacia una era ecozoica -donde lo ecológico sea el eje y así disminuir la devastación en curso-. Y para ello, no basta con el compromiso de los gobiernos ni sus autoridades, sino que con el compromiso de todos por medio de un "podercon" y "poder-del-interior", como planteaba Primavesi. En cierto modo, volver a reencontrarnos en comunidad con otros y otras, pero también con nosotros mismos, con el conocimiento de nuestro cuerpo y la conexión con la naturaleza.

Un abordaje desde el ecofeminismo permitiría reformular y ampliar las nociones conocidas de teología política. En primer lugar, el eje no se situaría exclusivamente en la soberanía estatal. Permitiría cuestionar la idea de poder y autoridad, situándose en la relación con el cosmos y nuevas formas de pensar el poder. En segundo lugar, las experiencias de las mujeres han permitido reafirmar la importancia de resignificar el rol de los cuerpos. En ese sentido, un cuerpo apropiado por ellas mismas para su placer, así como para la realización de ritos. Ambos términos que, en una comprensión de soberanía teológica-política, han sido apropiados por el Estado o la Iglesia, desde una visión ecofeminista logran visibilizar la importancia de conectarse con sí misma por medio del poder-delinterior o de la llamada Diosa. En tercer lugar, el ejemplo de Con-spirando en su anhelo de constituir un espacio alternativo, promueve lo que Mohanty plantea como "pedagogías de discrepancias" (pedagogies of dissent). Es decir, como un espacio que desafía espacios de dominación -tales como el antropocentrismo y androcentrismo-, para disputar espacios democráticos e inclusivos de género desde una visión ecofeminista.

Es por ello que, en un mundo post-secular, la superación de la noción antropocéntrica y androcéntrica debiera estar en el eje de las nuevas propuestas 
de teologías políticas y sus lecturas. Solo así podremos hablar de una teología política desde la resistencia no solo a la noción de soberanía, sino que integre también aquellas voces y posiciones que no están incorporadas en la discusión ni comprensión de lo teológico político. En ese sentido, comprender la teología política como un planteamiento no necesariamente dominante, sino que plantea alternativas desde la periferia y la rebeldía.

En este ensayo, se pretendió mostrar una posibilidad a ello, mediante la discusión, visibilización y lectura de autoras que escriben desde la experiencia y conocimiento de Latinoamérica. Pero también, desde la experiencia particular de Con-spirando como un ejemplo de política de "disidencia" desde una mirada ecofeminista. No obstante, este conocimiento aún no es ampliamente difundido en los círculos académicos. Por ello, y sumándome a las palabras de Mohanty $(2005,170)$, la academia en tanto espacio público para el diálogo, compromiso y visión de la democracia y justicia necesita del feminismo como proyecto político y pedagógico. Pero no solo ello. Hacer teología política desde el hemisferio sur o desde los Dos-Tercios significaría darle sentido a estos discursos para una comprensión de nuestra realidad sociopolítica y del planteamiento de nuevos modos de lo que significa el poder y el conocimiento de nuestros cuerpos. Por el otro lado, es una invitación para quienes leen el feminismo y el ecologismo como dos caminos separados sin encontrar punto de unión, ya que son más los caminos que unen que aquellos que nos distancian. 


\section{BIBLIOGRAFIA}

Agamben, Giorgio. 1995. Homo sacer. Il potere sovrano e la nuda vita. Torino: Einaudi.

Agamben, Giorgio. 1998. Quel che resta di Auschwitz. L'archivio e il testimone. Homo sacer. III. Torino: Bollati Boringhieri.

Agamben, Giorgio. 2003. Stato di Eccezione. Homo sacer II, 1. Torino: Bollati Boringhieri.

Agamben, Giorgio. 2007. Il regno e la gloria. Per una genealogia teologica dell'economia e del governo. Homo sacer II, 2. Vicenza: Neri Pozza.

Agamben, Giorgio. 2008. Il sacramento del linguaggio. Archeologia del giuramento. Homo sacer II, 3. Roma-Bari: Laterza.

Agamben, Giorgio. 2011. Altissima povertà. Regole monastiche e forma di vita. Homo sacer IV, 1. Vicenza: Neri Pozza.

Agamben, Giorgio. 2012. Opus Dei. Archeologia dell'ufficio. Homo sacer II, 5. Torino: Bollati Boringhieri.

Agamben, Giorgio. 2014. L'uso dei corpi. Homo sacer IV, 2. Vicenza: Neri Pozza.

Agamben, Giorgio. 2015. Stasis. La guerra civile come paradigma politico. Homo sacer II, 2. Torino: Bollati Boringhieri.

Benjamin, Walter. 1999. La dialéctica en suspenso. Fragmentos sobre la historia. Trad. Pablo Oyarzún. Santiago: LOM.

Casanova, José. 1994. Public Religions in the Modern World. Chicago: University of Chicago.

Christ, Carol P. 1994. "Por qué las mujeres necesitan a la Diosa: reflexiones fenomenológicas, sicológicas y políticas". En Ress, Mary Judith, Ute Seibert y Lene Sjørup (eds). 1994. Del cielo a la tierra. Una antología de teología feminista, 159-173. Santiago: Sello Azul.

Collingwood-Selby, Elizabeth. 1997. Walter Benjamin. La lengua del exilio. Santiago: LOM.

Collingwood-Selby, Elizabeth. 2009. El filo fotográfico de la historia. Walter Benjamin y el olvido de lo inolvidable. Santiago: Ediciones Metales Pesados.

Dotti, Jorge. 2000. Carl Schmitt en Argentina. Rosario: Homo sapiens editores.

Dotti, Jorge y Julio Pinto (comp.). 2002. Carl Schmitt, su época y pensamiento. Buenos Aires: Eudeba.

Gebara, Ivone. 2000. Intuiciones ecofeministas. Ensayo para repensar el conocimiento y la religión. Madrid: Trotta.

Gebara, Ivone. 2002. El rostro oculto del mal. Una teología desde la experiencia de las mujeres. Madrid: Trotta.

Habermas, Jürgen. 2008. "Secularism's Crisis of Faith: Notes on Post-Secular Society". New perspectives quarterly 25: 17-29. 
Karmy, Rodrigo (ed.). 2011. Políticas de la interrupción. Ensayos sobre Giorgio Agamben. Santiago: Editorial Escaparate.

Karmy, Rodrigo. 2014. Políticas de la ex-carnación. Para una genealogía teológica de la biopolítica. Buenos Aires: Editorial UNIPE.

Karmy, Rodrigo y Luna Follegati (eds.). 2018. Estudios en Gubernamentalidad. Ensayos sobre poder, vida y neoliberalismo. Viña del Mar: Ediciones Comunes.

Kateusz, Ally. 2019. Mary and Early Christian Women. Hidden Leadership. London: Palgrave MacMillan.

Mahmood, Saba. 2005. Politics of Piety: The Islamic Revival and the Feminist Subject. Princeton, N.J.: Princeton UP.

Mohanty, Chandra Talpade. 2003. Feminism without Borders: Decolonizing Theory, Practicing Solidarity. Durham: Duke UP.

Oro, Luis. 2005. "Crítica de Schmitt al liberalismo". Estudios públicos 98 (otoño): 171-187.

Oro, Luis. 2013. El concepto de realismo político. Santiago: RIL Editores.

Orrego, Ely. 2008. "Homo sacer y violencia divina en el caso judío: Lo insacrificable sometido a castigo". Revista Pléyade 2: 22-32.

Orrego, Ely. 2011. "Teología política: El nuevo paradigma de la soberanía y poder". Revista Pléyade 8: 1-6.

Primavesi, Anne. 1994. "Poder jerárquico y poder ecológico". En Ress, Mary Judith, Ute Seibert y Lene Sjørup (eds). Del cielo a la tierra. Una antología de teología feminista, 455-479. Santiago: Sello Azul.

Ress, Mary Judith, Ute Seibert y Lene Sjørup (eds). 1994. Del cielo a la tierra. Una antología de teología feminista. Santiago: Sello Azul.

Ress, Mary Judith. 2006. Ecofeminism in Latin America. New York: Orbis Books, Maryknoll.

Ress, Mary Judith. 2012. Sin visiones nos perdemos. Reflexiones sobre Teología Ecofeminista Latinoamericana. Santiago: Colectivo Con-spirando.

Richard, Nelly. 1994. "Roturas, memoria y discontinuidades (en homenaje a W. Benjamin)". En La insubordinación de los signos, 13-36. Santiago de Chile: Cuarto Propio.

Richard, Nelly. 1999. "Memoria del arte y traza fotográfica". Revista Teoría del Arte: 37-46. Recuperado de: https://bit.ly/2N4r9U87

Richard, Nelly. 2018. "Crítica Cultural". Centro Nacional de Arte Contemporáneo. Recuperado de: https://bit.ly/2Zzpjfs

Schmitt, Carl. 2009. Teología política. Madrid: Trotta.

Scott, Joan Wallach. Sex and Secularism. Princeton, New Jersey: Princeton UP, 2018

Shiva, Vandana, y Maria Mies. 2014. Ecofeminism. Londres y Nueva York: Zed Books.

Stone, Merlin. 1994. "Cuando Dios era mujer". En Ress, Mary Judith, Ute Seibert y Lene Sjørup (eds). Del cielo a la tierra. Una antología de teología feminista, 175-184. Santiago: Sello Azul. 
Taylor, Charles. 2007. A Secular Age. Cambridge, Mass.: Belknap of Harvard UP.

Thayer, Willy. 2007. "El giro barroco. De G. Deleuze a W. Benjamin". Archivos. Revista de Filosofía 2/3: 93-119.

Thayer, Willy. 2010. Tecnologías de la crítica: Entre Walter Benjamin y Gilles Deleuze. Santiago: Ediciones Metales Pesados.

van Andel, Arianne. 2014. "Entre lucha política y relacionalidad cosmológica: La contribución de Ivone Gebara a la teología de la liberación". Mandrágora 20 (20): 7-24.

Vatter, Miguel. 2004. "Strauss and Schmitt as Readers of Hobbes and Spinoza. On the Relation between Liberalism and Political Theology". The New Centennial Review 4 (3): 161-214.

Vatter, Miguel. 2009. "Political Theology Without Sovereignty: Some 20th Century Examples (Voegelin, Maritain, Badiou)" (August 26). Recuperado de: https://bit.ly/2WWnfBi

Vatter, Miguel (ed.). 2011a. Crediting God: Sovereignty \& Religion in the Age of Global Capitalism. New York: Fordham University Press.

Vatter, Miguel. 2011b. "Habermas between Athens and Jerusalem: Public Reason and Atheistic Theology". Interpretation: A Journal of Political Philosophy 28 (3): 243 -261.

Vatter, Miguel. 2011c. "Pensar la política desde la teología política (entrevistado por Ely Orrego). Revista Pléyade 8: 185-198.

Vatter, Miguel. 2012. Constitución y resistencia. Ensayos de teoría democrática radical. Santiago: Ediciones Universidad Diego Portales.

Vatter, Miguel. 2013. "Politico-Theological Foundations of Human Rights, The Case of Maritain". Social Research: An International Quarterly, 80 (1): 233-260.

Vatter, Miguel. 2016. "Cosmopolitan Political Theology in Cohen and Rosenzweig". Philosophy Today 60 (2): 295-324.

Vatter, Miguel. 2019a. "'Only a God Can Resist a God' Political Theology between Polytheism and Gnosticism". Political Theology DOI: 10.1080/1462317X.2019.1618597

Vatter, Miguel. 2019b. "Civil Religion and the Pursuit of Happiness from Machiavelli to Italian Theory". Giornale Critico Di Storia Delle Idee 1: 7589. 